\title{
Adipose-Derived Mesenchymal Stem Cells for the Treatment of Knee Osteoarthritis: A Systematic Review
}

\author{
Milani $L^{1 *}$ and Ferrari $\mathbf{S}^{2}$ \\ ${ }^{1}$ Department of Trauma and Orthopaedic Surgery, \\ Maggiore Hospital, Largo Nigrisoli 2, Bologna, Italy \\ ${ }^{2}$ Department of Morphology, Surgery and Experimental \\ Medicine, University of Ferrara, Ferrara, Italy \\ *Corresponding author: Milani L, Department of \\ Trauma and Orthopaedic Surgery, Maggiore Hospital, \\ Largo Nigrisoli 2, 40133 Bologna, Italy
}

Received: September 16, 2021; Accepted: October 13, 2021; Published: October 20, 2021

\begin{abstract}
Purpose: For several years, adipose tissue has gained increasing interest as an ideal source of mesenchymal stem cells for the regenerative treatment of numerous pathologies and degenerative processes, as like Knee Osteoarthritis (KOA). The aim of our study was to report postoperative clinical, functional and radiological outcomes in patients with KOA treated with intra-articular injection of autologous Adipose-Derived Stem Cells (ADSCs).
\end{abstract}

Methods: We performed a systematic review searching for all the clinical studies dealing with the use of ADSCs for the treatment of KOA published in PubMed and Embase until April 2021. We included 8 prospective studies dealing with postoperative outcomes after ADSCs therapy.

Results: All clinical and functional outcomes improved after intra-articular injections of ADSCs. Postoperative magnetic resonance imaging (MRI) scores showed an increased quality of repaired cartilage compared to the preoperative time. No serious advent events were observed.

Conclusion: This study suggests that ADSCs therapy seems to be safe and effective. It can be considered an innovative procedure for improved cartilage regeneration and as adjuvant for the surgical treatment of diffuse degenerative chondral lesions with good clinical and radiological outcomes.

Keywords: Adipose-derived stem cells; Knee osteoarthritis; Clinical outcomes; Radiological outcomes

\section{Abbreviations}

KOA: Knee Osteoarthritis; MRI: Magnetic Resonance Imaging; ADSCs: Stem Cells taken from the Adipose Tissue; WOMAC: Knee Injury and Osteoarthritis Outcomes Score and Western Ontario and McMaster Universities Osteoarthritis Index; NPRS: Numeric Pain Rating Scale; KOOS: Knee Injury and Osteoarthritis Outcome Score; IKDC: International Knee Documentation Committee Score; IKS: International Knee Society Knee and Function Scores; ROM: Range of Motion; EQOL: Emory Quality of Life questionnaire; SF-36: ShortForm 36; SAS: Short Arthritis Assessment Scale; ICRS: International Cartilage Repair Society; MOAKS: MRI Osteoarthritis Knee Scores; VAS: Visual Analogue Pain Scale

\section{Introduction}

Knee Osteoarthritis (KOA) is a degenerative condition that is often unresponsive to conservative treatments, such as nonpharmacological interventions, systemic drug treatment and intraarticular therapies. Therefore, a sustainable approach is represented by regenerative medicine with stem cells for managing of early stages (mild and moderate KOA) [1].

Specifically, the stem cells that can differentiate into chondrocytes are the mesenchymal ones, namely ADSCs (Stem Cells taken from the Adipose Tissue), whose elective source is adipose tissue because it is easy to collect and is always available [2]. Because of their multilineage potential, limited immunogenicity, immunosuppressive activities, and relative ease to growth in culture, the mesenchymal stem cells have attracted attention for clinical use. The ability to differentiate into chondrocytes makes stem cells therapy regenerative because, once injected near the lesion, they differentiate into new cells that replace obsolete or damaged ones by renewing the tissues [3].

Precisely, these cells showed a special and specific regenerative activity towards the cartilage tissue, intended to create a solid but flexible scaffolding and seem to decelerate the progression of knee osteoarthritis [4].

The aim of our study was to report postoperative clinical, functional and radiological outcomes in patients with knee osteoarthritis treated with intra-articular injection of adipose-derived stem cells.

\section{Materials and Methods}

We performed a systematic review following the PRISMA guidelines. We searched and included all prospective studies dealing with postoperative clinical, functional and radiological outcomes after the treatment of KOA with intra-articular knee injection of ADSCs published in PubMed and Embase until March 2021 using the keyword "stem cells" OR "derived stem cells" OR "adipose derived stem cells" OR "mesenchymal cells" AND "knee" OR "osteoarthritis".

We initially identified 195 records. We excluded reviews, letters to the editor, biomechanical and histological studies, cadaveric and animal studies. After examination of titles and abstracts, we finally included in our systematic review 8 prospective studies [5-12] (Figure $1)$. 


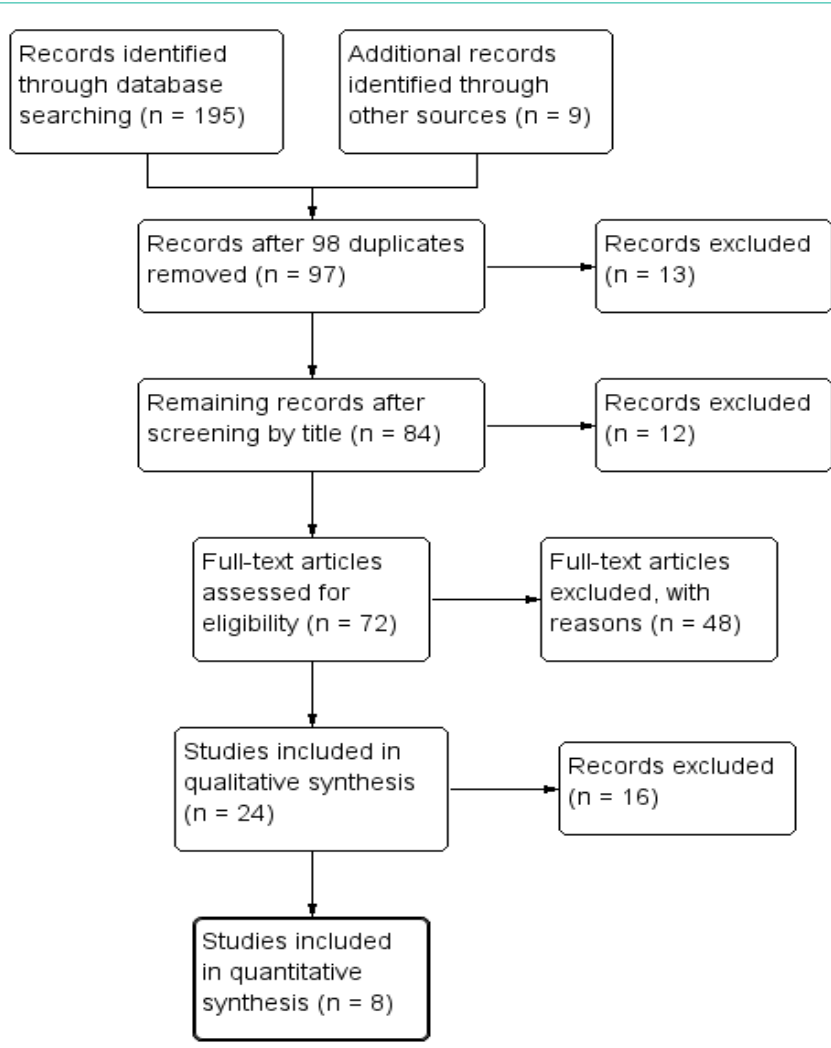

Figure 1: Flow-chart of the reviewed studies according to PRISMA guidelines.

One study [6] compared postoperative outcomes of a group of patients performed ADSCs therapy with another control group without this type of regenerative treatment. One study [5] compared outcomes of a group of patients performed a single injection with another one performed two injections of ADSCs. One study [9] compared outcome of a group of patients performed ADSCs therapy with another control group treated with injection of normal saline. Another study [10] compared outcome of patients performed ADSCs therapy with another control group treated with bone marrow aspirate concentrate injection.

Clinical and functional outcomes were evaluated using the Knee Injury and Osteoarthritis Outcomes Score and Western Ontario and McMaster Universities Osteoarthritis Index (WOMAC) score $[5,9,11,12]$, the Numeric Pain Rating Scale (NPRS) [5], the Knee Injury and Osteoarthritis Outcome Score (KOOS) [5,10-12], the International Knee Documentation Committee (IKDC) score [6], the Lysholm score $[6,8,11]$, the International Knee Society (IKS) knee and function scores [6], the Visual Analogue pain scale (VAS) [6,7,9-12], the Range of Motion (ROM) [9], the IKDC-subjective [11], the Tegner activity scale [8], the Emory Quality of Life (EQLS) questionnaire [10], the Short-Form 36 (SF-36) [12] and the Short arthritis assessment scale (SAS) [12].

An arthroscopic second-look surgery was performed at a mean of 12.4 months postoperatively to evaluate cartilage lesions and regeneration using the International Cartilage Repair Society (ICRS) score $[6,7,12]$.

Radiological outcomes were assessed in 4 studies using postoperative magnetic resonance imaging (MRI) [5,7,9,12]. MRI Osteoarthritis Knee Scores (MOAKS) [5] were used to describe osteoarthritis progression over time and to evaluate changes of cartilage defects after injection as previously published [13].

Inclusion criteria for surgery were patients aged 18 years and older $[5,8,9,12]$ with unilateral moderate symptomatic knee pain $[5,6,12]$ and Grade II-III medial or lateral compartment knee osteoarthritis according to the modified Kellgren-Lawrence classification $[5,6,8,9,12]$, symptomatic knee osteoarthritis and failure of conservative treatments after three [6], six or more months [7].

All patients included had previously attempted primary conservative management of knee osteoarthritis using simple analgesia, exercises and weight management program and biomechanical adjustment with bracing and orthotics if relevant $[5,6]$.

Exclusion criteria for surgery were patients with a recent traumatic event to the knee with meniscal tears or ligament lesions [5-8], knee instability [6], villonodular synovitis or chondromatosis of the knee [7], previous intra-articular injectable therapies within the last 3-6 months $[5,8,12]$, previous arthroscopic treatment for KOA [8], joint infection $[6,7,8]$, metabolic or inflammatory arthritis $[6,8,12]$, varus or valgus deformity of $5^{\circ}$ or more [8], pregnancy $[5,7,8]$, breast feeding [5], malignancy [5,7,12], patients with coagulation and bleeding disorders [5,7], persons over 70 years of age [8], a major neurological deficit [8] and serious medical illness (life expectancy of $<2$ years or a high intraoperative risk) [8].

The surgical procedure was performed with patient in a supine position on the operating table $[6,7]$ under spinal anesthesia, with the use of a tourniquet $[6,8]$. Firstly, using a lateral abdominal approach [5], a percutaneous infiltration with saline solution mixed with ropivacaine/lidocaine and adrenaline was performed [5,7]. Fifteen minutes later, it was completed the abdominal subcutaneous fat aspiration with a standard lipoaspiration technique $[5,7,9]$. In one study [8] the adipose synovium was harvested from the inner side of the infrapatellar fat pad by the extension of the skin incision at the arthroscopic lateral portal side. After the harversting of adipose tissue, the mature adipocytes were separated from the stromal vascular fraction by centrifugation [9] as described by Zuk et al. [14] and using enzymatic digestion with collagenase and later cell culturing under hypoxic condition [5,9].

The harvested micro-fragmented adipose tissue was introduced into the Lipogems ortho kit (Lipogems International SpA, Milan, Italy) [7]. After isolation, the ADSCs represented a mean of $9.4 \%$ (range 8.2-10.9\%) of the stromal vascular fraction cells [6]. Associated procedures included arthroscopic debridement and synovectomy in 127 patients in 3 studies [6,7,8], 8 meniscectomy, 3 high tibial osteotomy, 2 microfractures and 2 ACL/LCL reconstructions in 1 study [11].

At the end of the arthroscopic procedure, the microfragmented adipose tissue products were injected intra-articularly in the medial knee space under arthroscopic guidance [5-7,9].

\section{Results}

Demographic data are reported in Table 1. A total of 212 patients with knee osteoarthritis were included in this systematic review. All 
Table 1: Demographic data of included studies.

\begin{tabular}{|c|c|c|c|c|c|}
\hline Authors & N. patients & Gender (male/female) & Mean age of patients (range) & Mean BMI kg/m² (range) & Mean follow-up Months (range) \\
\hline Freitag et al. [5] & 20 & $11 / 9$ & $54.7(45-65)$ & $30.4(24.8-36)$ & $12.0(\mathrm{~N} / \mathrm{A})$ \\
\hline Kim et al. [6] & 50 & $16 / 34$ & $59.2(53-65)$ & $26.6(22.2-31.7)$ & $37.2(36-42)$ \\
\hline Panni et al. [7] & 52 & $22 / 30$ & $67.3(37-78)$ & $28.1(21-34)$ & $15.3(6-24)$ \\
\hline Koh et al. [8] & 25 & $8 / 17$ & $54.2(45-64)$ & $\mathrm{N} / \mathrm{A}$ & $16.4(12-18)$ \\
\hline Lee et al. [9] & 12 & $3 / 9$ & $62.2(56-69)$ & $25.3(20.4-30.2)$ & $6.0(\mathrm{~N} / \mathrm{A})$ \\
\hline Mautner et al. [10] & 35 & $12 / 23$ & $63.0(52-74)$ & N/A & $12.9(7-19)$ \\
\hline Russo et al. [11] & 30 & $18 / 12$ & $44.7(33-56)$ & $25.9(22.6-29.2)$ & $36.0(N / A)$ \\
\hline Pers et al. [12] & 18 & $8 / 10$ & $64.6(56-72)$ & $27.6(24.5-30.7)$ & $6.0(\mathrm{~N} / \mathrm{A})$ \\
\hline
\end{tabular}

N/A: Not Available; BMI: Body Mass Index.

Table 2: Outcomes, complications and adverse events.

\begin{tabular}{|c|c|c|c|}
\hline Authors & Preoperative clinical outcomes (mean) & Postoperative clinical outcomes (mean) & Complications and adverse events \\
\hline Freitag et al. [5] & $\begin{array}{c}\text { NPRS: } 6.5 \\
\text { KOOS: } 55 \\
\text { WOMAC: } 54.4\end{array}$ & $\begin{array}{c}\text { NPRS: } 2.3 \\
\text { KOOS: } 80 \\
\text { WOMAC: } 87.3\end{array}$ & none \\
\hline Kim et al. [6] & $\begin{array}{c}\text { IKDC: } 36.5 \\
\text { Lysholm score: } 55.7\end{array}$ & $\begin{array}{c}\text { IKDC: } 64.8 \\
\text { Lysholm score: } 84.7\end{array}$ & none \\
\hline Panni et al. [7] & $\begin{array}{c}\text { IKS knee score: } 37.4 \\
\text { IKS function score: } 57.2 \\
\text { VAS: } 8.5\end{array}$ & $\begin{array}{l}\text { IKS knee score: } 62.6 \\
\text { IKS function score: } 83.0 \\
\text { VAS: } 5.1\end{array}$ & 3 abdominal transitory hematoma \\
\hline Koh et al. [8] & $\begin{array}{c}\text { Lysholm score: } 41.2 \\
\text { Tegner activity scale: } 1.5 \\
\text { VAS: } 4.9\end{array}$ & $\begin{array}{c}\text { Lysholm score: } 68.1 \\
\text { Tegner activity scale: } 2.8 \\
\text { VAS: } 2.7\end{array}$ & 1 pain and swelling after injection \\
\hline Lee et al. [9] & $\begin{array}{l}\text { WOMAC: } 60.0 \\
\text { VAS: } 6.8 \\
\text { ROM: } 127.9^{\circ}\end{array}$ & $\begin{array}{l}\text { WOMAC: } 26.7 \\
\text { VAS: } 3.4 \\
\text { ROM: } 134.6^{\circ}\end{array}$ & $\begin{array}{c}6 \text { arthralgia; } \\
2 \text { joint effusion }\end{array}$ \\
\hline Mautner et al. [10] & $\begin{array}{c}\text { VAS: } 4.3 \\
\text { EQOL: } 0.66 \\
\text { KOOS pain: } 51.4\end{array}$ & $\begin{array}{c}\text { VAS: } 2.8 \\
\text { EQOL: } 0.77 \\
\text { KOOS pain: } 70.4\end{array}$ & none \\
\hline Russo et al. [11] & $\begin{array}{c}\text { KOOS: } 58 \\
\text { VAS: } 5.8 \\
\text { Lysholm score: } 48 \\
\text { IKDC subjective: } 35\end{array}$ & $\begin{array}{c}\text { KOOS: } 80 \\
\text { VAS: } 2.8 \\
\text { Lysholm score: } 75 \\
\text { IKDC subjective: } 60\end{array}$ & 7 additional treatments \\
\hline Pers et al. [12] & $\begin{array}{l}\text { WOMAC: } 60.7 \\
\text { KOOS: } 34.0 \\
\text { VAS: } 77 \\
\text { SAS: } 29 \\
\text { SF: } 36: 30.9\end{array}$ & $\begin{array}{l}\text { WOMAC: } 27.6 \\
\text { KOOS: } 65.8 \\
\text { VAS: } 35.8 \\
\text { SAS: } 17.7 \\
\text { SF: } 36: 39.1\end{array}$ & 4 slight knee pain/joint effusionn \\
\hline
\end{tabular}

WOMAC: Knee Injury and Osteoarthritis Outcomes Score and Western Ontario and McMaster Universities Osteoarthritis Index; KOOS: Knee Injury and Osteoarthritis Outcome Score; NPRS: Numeric Pain Rating Scale; EQOL: Emory Quality of Life Questionnaire; SAS: Short Arthritis Assessment Scale; SF-36: Short-Form 36; IKDC: International Knee Documentation Committee score; ROM: Range of Motion; VAS: Visual Analogue Pain Scale; IKS: International Knee Society knee and function scores.

studies reported the gender: 98 were males (40.5\%) while 144 were females (59.5\%). The mean age of patients was 57.9 (range 33-78). The mean BMI was 27.3 (range 20.4-36), while the mean follow-up was 17.7 months (range 6-42).

All clinical and functional outcomes (KOOS, WOMAC, NPRS, IKDC, IKS, ROM, Lysholm score, VAS, SAS, SF-36, Tegner activity scale, EQOL) improved after intra-articular injection of ADSCs in all included studies [5-9] (Table 2). In particular, in one study [5] postoperative KOOS, WOMAC and NPRS significantly improved in the group of patients performed ADSCs therapy compared to a control group without this regenerative treatment. Moreover, in another study [6] postoperative IKDC and Lysholm score improved more in the group of patients performed two injections than the group of patients with a single injection of ADSCs.

At arthroscopic second-look surgery [6], ICRS grades significantly improved in the injection group than in the control group as an index of increased quality of repaired cartilage.
No serious adverse events were observed in any included study. A total number of complications identified in this present review is $27 / 212(12.7 \%)$, most of which were minor. A transitory haematoma of the abdominal region that have not influenced the post-operative knee recovery in 3 patients in 1 study [7]. Pain and swelling after the injection, which resolved spontaneously after 2 weeks or with nonsteroidal anti-inflammatory drugs were noted in 5 patients in 2 studies [8,12]. Arthralgia was noted in 6 patients in 1 study [9]. Joint effusion was noted in 2 patients in 1 study [9]. Minor discomfort and bruising were noted in 4 patients in 1 study [5].

In one study [11] 7 patients (23\%) received additional treatments in the period of observation, and therefore have been considered failures: one patient received 3 injections of hyaluronic acid, while 6 patients had multiple injections of platelet rich plasma.

Over $90 \%$ of patients were satisfied with clinically significant pain and functional improvement noted in 4 studies at the postoperative follow-up at 6 months [9] and 1 year $[5,7,8]$. 
Based on MRI Osteoarthritis Knee Scores (MOAKS) analysis and on the evaluation of the size of the cartilage defects according to the modified Noyes grading system [15], almost all patients had improvement in cartilage or no progression in cartilage loss and in osteophyte formation at 6 months [7,9,12] and 12 months [5] of follow-up. In particular, Freitag et al. [5] reported a total of $67 \%$ of participants within the control group had progression of cartilage loss with a further $56 \%$ having extension of osteophyte formation. By comparison, in the one-injection group only $30 \%$ of participants had further cartilage loss although $50 \%$ had progression of osteophyte formation at 12 months. In the two-injection group, 89\% of participants had improvements in cartilage or no progression in cartilage loss with stabilization of KOA also indicated by $89 \%$ having no progression in osteophyte formation.

Lee et al. [9] showed the size of the cartilage defect in MRI at 6 months was not significantly changed in the ADSCs group, whereas the size of the cartilage defect in the control group was significantly increased. In addition, there was a significant difference between the 2 groups in the amount of change in cartilage defect after the injection.

\section{Discussion}

Intra-articular injection of ADSCs seems to be a safe and effective treatment for knee osteoarthritis and may have the ability to prevent disease progression [5]. In fact, no serious adverse events were observed during the follow-up period, showing the safety profile of this surgical procedure. Previous preclinical studies had already successfully shown benefit of intra-articular ADSCs therapy in observed pain and functional improvement $[16,17]$. Preliminary studies in goats and rabbits showed that cartilage regeneration did not occur at the expense of chondrogenic differentiation of the injected cells but may be strongly related to a secondary stimulation of endogenous progenitor cells through paracrine effects [18].

Four studies $[5,6,9,12]$ reported no progression of KOA basing on postoperative MRI and with MRI Osteoarthritis Knee Scores (MOAKS) analysis at the final follow-up. Similarly, past studies had indicated the ability of intra-articular ADSCs therapy to modify the progression of KOA. Jo et al. [20] reported increased cartilage volume at 6 months following ADSCs therapy and a later study using allogenic bone marrow derived mesenchymal stem cells successfully stabilizing KOA and improving cartilage quality [21].

However, this systematic review also highlighted that some included studies performed surgical procedures in association with stem cell therapy, which may have positively influenced the clinical outcomes. In particular, associated procedures such as arthroscopic debridement and synovectomy [6-8] had increased the overall midterm outcomes of patients with early KOA in 3 studies [6-8]. This was in lineage with reported by Cui et al. in their meta-analysis [19], including 18 clinical trials on the efficacy of ADSCs, where had observed that patients with arthroscopic debridement and lower degrees of Kellgren-Lawrence KOA obtained higher clinical and functional outcomes.

In our study, we found a complication rate of ADSCs therapy equal to $12.7 \%$ while no serious adverse events. In literature it was reported the complication rate of the liposuction procedure is very low, about $0.1 \%$ according to a national survey of 112.756 reported patient procedures [22]. Regarding the complication rate and incidence of adverse events after treatment with stem cells, a multicenter analysis performed among 2372 patients undergoing autologous stem cell therapy for different orthopaedic conditions revealed that these procedures are safe: the incidence of adverse events was of $12.1 \%$ (the majority were pain and knee swelling), the incidence of serious adverse events was $1.5 \%$ (neoplasm, neurologic and vascular events), the incidence of neoplasm was $0.3 \%$ (in contrast, the annual incidence of cancer in United States population in 2011 was $0.44 \%$ ) [23].

\section{Conclusion}

ADSCs therapy can be considered an innovative procedure for improved cartilage regeneration and as adjuvant for the surgical treatment of diffuse degenerative chondral lesions with good clinical and radiological outcomes. The results of this study demonstrated that intra-articular injection of ADSCs is simple, affordable, minimally invasive and provides assistance in reducing pain and improving function in patients with KOA.

\section{Declaration}

Ethics approval: all procedures performed in studies involving human participants were in accordance with the ethical standards of the institutional and national research committee and with the 1964 Helsinki Declaration and its later amendments or comparable ethical standards. The study was approved by the Bioethics Committee "Area Vasta Emilia Centro" (CE-AVEC) of the Medical University of Bologna.

Consent to participate: Informed consent was obtained from all individual participants included in the study.

Consent for publication: Patients signed informed consent regarding publishing their data and photographs.

\section{References}

1. Caplan Al. Adult mesenchymal stem cells: when, where, and how. Stem Cells Int. 2015; 2015: 628767.

2. Nakagami H, Morishita R, Maeda K, Kikuchi Y, Ogihara T, Kaneda Y. Adipose tissue-derived stromal cells as a novel option for regenerative cell therapy. J Atheroscler Thromb. 2006; 13: 77-81.

3. Centeno C, Busse D, Kisiday J, Keohan C, Freeman M, Karli D. Increased knee cartilage volume in degenerative joint disease using percutaneously implanted, autologous mesenchymal stem cells. Pain Physician. 2008; 11: 343-353.

4. Lalu MM, McIntyre L, Pugliese C, Fergusson D, Winston BW, Marshall JC, et al. Safety of cell therapy with mesenchymal stromal cells (safe cell): a systematic review and meta-analysis of clinical trials. PLoS One. 2012; 7 : e47559.

5. Freitag J, Bates D, Wickham J, Shah K, Huguenin L, Tenen A, et al. Adiposederived mesenchymal stem cell therapy in the treatment of knee osteoarthritis: a randomized controlled trial. Regen Med. 2019; 14: 213-230.

6. Kim YS, Koh YG. Comparative matched-pair analysis of open-wedge high tibial osteotomy with versus without an injection of adipose-derived mesenchymal stem cells for varus knee osteoarthritis: clinical and secondlook arthroscopic results. Am J Sports Med. 2018; 46: 2669-2677.

7. Panni AS, Vasso M, Braile A, Toro G, De Cicco A, Viggiano D, et al. Preliminary results of autologous adipose-derived stem cells in early knee osteoarthritis: identification of a subpopulation with greater response. Int Orthop. 2019; 43: 7-13.

8. Koh YG, Choi YJ. Infrapatellar fat pad-derived mesenchymal stem cell therapy for knee osteoarthritis. Knee. 2012; 19: 902-907. 
9. Lee WS, Kim HJ, Kim KI, Kim GB, Jin W. Intra-articular injection of autologous adipose tissue-derived mesenchymal stem cells for the treatment of knee osteoarthritis: a phase Ilb, randomized, placebo-controlled clinical trial. Stem Cells Transl Med. 2019; 8: 504-511.

10. Mautner K, Bowers R, Easley K, Frausel Z, Robinson R. Functional outcomes following micro fragmented adipose tissue versus bone marrow aspirate concentrate injections for symptomatic knee osteoarthritis. Stem Cells Trans Med. 2019; 8: 1149-1156.

11. Russo A, Screpis D, Di Donato SL, Bonetti S, Piovan G, Zorzi C. Autologous micro-fragmented adipose tissue for the treatment of diffuse degenerative knee osteoarthritis: an update at 3 year follow-up. J Exp Orthop. 2018; 5: 52.

12. Pers YM, Rackwitz L, Ferreira R, Pullig O, Delfour C, Barry F, et al. Mesenchymal stromal cell-based therapy for severe osteoarthritis of the knee: a phase I dose-escalation trial. Stem Cells Transl Med. 2016; 5: 847 856.

13. Runhaar J, Schiphof D, van Meer B, Reijman M, Bierma-Zeinstra SMA Oei EHG. How to define subregional osteoarthritis progression using semiquantitative MRI Osteoarthritis Knee Score (MOAKS). Osteoarthr Cartil. 2014; 22: 1533-1536.

14. Zuk PA, Zhu M, Mizuno H, Huang J, Futrell JW, Katz AJ, et al. Multilineage cells from human adipose tissue: implications for cell-based therapies. Tissue Eng. 2001; 7: 211-228.

15. Kijowski R, Blankenbaker DG, Davis KW, Shinki K, Kaplan LD, De Sme AA. Comparison of 1.5- and 3.0-T MR imaging for evaluating the articular cartilage of the knee joint. Radiology. 2009; 250: 839-848.

16. Black L, Gaynor J, Adams C, Dhupa S, Sams AE, Taylor R, et al. Effect of intra-articular injection of autologous adipose-derived mesenchymal stem and regenerative cells on clinical signs of chronic osteoarthritis of the elbow joint in dogs. Vet Ther. 2008; 9: 192-200.

17. Lee KB, Hui JH, Song IC, Ardany L, Lee EH. Injectable mesenchymal stem cell therapy for large cartilage defects - a porcine model. Stem Cell. 2007; 25: 2964-2971.

18. Murphy JM, Fink DJ, Hunziker EB, Barry FP. Stem cell therapy in a caprine model of osteoarthritis. Arthritis Rheum. 2003; 48: 3464-3474.

19. Cui GH, Wang YY, Li CJ, Shi CH, Wang WS. Efficacy of mesenchymal stem cells in treating patients with osteoarthritis of the knee: a meta-analysis. Exp Ther Med. 2016; 12: 3390-3400.

20. Jo CH, Lee YG, Shin WH, Kim H, Chai JW, Jeong EC, et al. Intra-articular injection of mesenchymal stem cells for the treatment of osteoarthritis of the knee: a proof of concept clinical trial. Stem Cells. 2014; 32: 1254-1266.

21. Peeters CM, Leijs MJ, Reijman M, van Osch GJVM, Bos PK. Safety of intra-articular cell-therapy with culture-expanded stem cells in humans: a systematic literature review. Osteoarthr Cartil. 2013; 21: 1465-1473.

22. Teimourian B, Rogers WB. A national survey of complications associated with suction lipectomy: a comparative study. Plast Reconstr Surg. 1989; 84: 628-631.

23. Centeno CJ, Al-Sayegh H, Freeman MD, Smith J, Murrell WD, Bubnov R. A multicenter analysis of adverse events among two thousand, three hundred and seventy two adult patients undergoing adult autologous stem cell therapy for orthopedic conditions. Int Orthop. 2016; 40: 1755-1765. 